\title{
Congenital Eventration of Diaphragm - Diagnostic Dilemma in Blunt Thoracoabdominal Trauma
}

\author{
Villalan Ramasamy ${ }^{1}$, Benet Duraisamy ${ }^{2}$, Srinivasan Muthukrishnan ${ }^{3}$, \\ Rajendran. $S^{4}$, Naganath Babu.O.L ${ }^{4}$ \\ Institute Of Surgical Gastroenterology, Rajiv Gandhi Govt.General Hospital, Madras Medical College \\ ,Dr.M.G.R.Medical University, Chennai ,Tamilnadu ,India.
}

\begin{abstract}
Eventration of diaphragm is defined as an abnormal, permanent elevation of the hemidiaphragm and its lax membrane is interspersed with the normal peritoneal and pleural membranes. Most adults with Eventration of diaphragm are asymptomatic and do not require surgical treatment .However the preexistence of this condition in cases of thoraco abdominal trauma mimics a traumatic rupture of diaphragm and hence, is a diagnostic dilemma. We present a case of previously undiagnosed Congenital Eventration of diaphragm mimicking a traumatic rupture of diaphragm.
\end{abstract}

Keywords: Congenital Eventration diaphragm, traumatic rupture

\section{Introduction}

Eventration of diaphragm is defined as an abnormal, permanent elevation of the hemidiaphragm and its lax membrane is interspersed with the normal peritoneal and pleural membranes. Most adults with Eventration of diaphragm are asymptomatic and do not require surgical treatment .However the preexistence of this condition in cases of thoraco abdominal trauma mimicks a traumatic rupture of diaphragm and hence, is a diagnostic dilemma. We present a case report of Congenital Eventration of diaphragm in a middle aged patient with thoraco abdominal trauma .

\section{Case Report}

49 years old male presented with history of Road traffic accident, sustaining injury to the chest and abdomen. Patient had difficulty in breathing and upper abdominal pain. There was no history of Loss of consciousness and No history of vomiting or seizures .After adequate analgesia patient's pulse, blood pressure and saturation were normal. On examination patient was conscious, oriented and had tenderness over the left lower ribs with Decreased breath sounds on the left side. Bowel sounds were heard on left lower side of the chest. Abdominal examination revealed a tender epigastrium, left hypochondrium and left lumbar region and localized guarding . Chest X ray showed elevated Left dome of the diaphragm (Fig 1). Ultrasonogram showed spleen in the left thoracic cavity with no perisplenic collection and bowel loops with normal peristalsis in the left thoracic cavity and a highly positioned Diaphragm. There was no diaphragmatic defect or free fluid. Abdomino Thoracic Computed tomography revealed a Left diaphragmatic eventration with bowel and spleen as contents with a normal Lung parenchyma( Fig 2). CT Pelvis showed a Right acetabular fracture with inferior pubic rami fracture. In view of the clinical and imaging findings this case was managed non operatively and close monitoring of vitals and serial clinical examination.

\section{Discussion}

Traumatic rupture of the diaphragm due to blunt trauma is a rare entity and occurs in less than $5 \%$ of all blunt abdominal and thoracic injuries. The diaphragmatic defect resulting from blunt trauma usually extends in a posterolateral fashion from the central tendon and is more common on the left as the left posterior leaf is weaker than the rest of the diaphragm. The prognosis of this condition depends on the timing of diagnosis, associated injuries and pre existing non traumatic disease [1]. Delayed diagnosis of traumatic rupture of the diaphragm leads to catastrophic complications due to the consequences of bowel incarceration and strangulation[2]. Eventration of diaphragm is a relatively rare entity and defined as high arched hemi diaphragm without discontinuity .Clinical features vary from asymptomatic to life threatening respiratory distress. Eventration is characterised by a atrophied and thin walled diaphragm mainly composed of fibrous tissue. In thoracoabdominal trauma ,preexisting Eventration of diaphragm is difficult to differentiate from a traumatic rupture which may result in a needless laparotomy which neverthless makes the diagnosis obvious [3]. Eventration of diaphragm can also be diagnosed as Pneumothoraces and hemothoraces resulting in the placement of chest tube.[4,5]. Therefore a thorough history taking and detailed examination of the diaphragmatic contour and continuity on imaging is paramount to institute appropriate management. When the 
imaging findings are inconclusive, diagnostic laparoscopy should be considered in patients who are hemodynamically stable[5]. Acute injuries are best approached through an abdominal incision. Patients who are operated on more than a week or two after the acute injury are best approached through the thoracic route.

\section{Conclusion}

This case demonstrates the need for a thorough history taking and physical examination along with a urgent chest radiograph and CT imaging to differentiate between a preexisting congenital eventration of diaphragm and a traumatic diaphragmatic rupture in cases of thoraco abdominal blunt trauma .This is because early identification and emergent surgery is warranted in a traumatic rupture whereas congenital eventration of diaphragm generally does not warrant emergency intervention.

\section{References}

[1]. Richard E.Ward.,Timothy C.Flynn.,William P.Clark. Diaphragmatic Disruption Secondary to Blunt Abdominal Trauma. The Journal of Trauma Vol. 21,No.1

[2]. W.J Lee.,Y.S.Lee .Traumatic Diaphragmatic rupture : a diagnostic challenge in the emergency department. BMJ Case Rep 2009

[3]. C.M .Moore,B.J.Mander,M.A.K.Raja . A case of congenital Eventration of the Diaphragm mimicking traumatic Diaphragmatic rupture. Int.J.Care Injured 32 (2001) 508-509

[4]. F.Bonanno . Eventration of the Diaphragm : A Pitfall in blunt Thoracic trauma Injury Extra 40 (2009)127-129

[5]. Mantoo S.K,Mak K. Congenital Diaphragmatic Eventration in an Adult : A Diagnostic dilemma. Singapore Med J 2007 ; 48(5):e 136

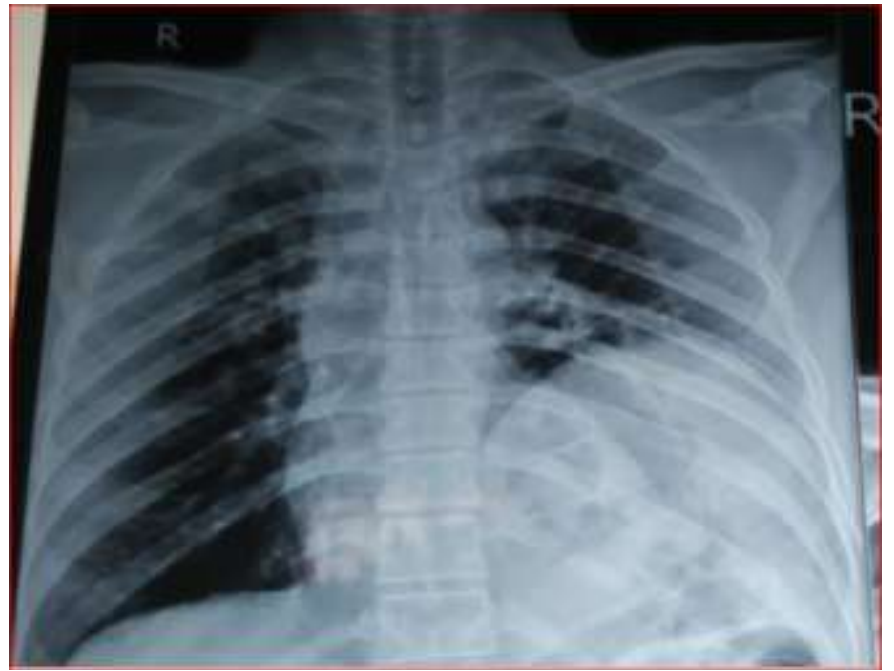

Fig .1 Chest X ray showing Elevated Left dome of the diaphragm

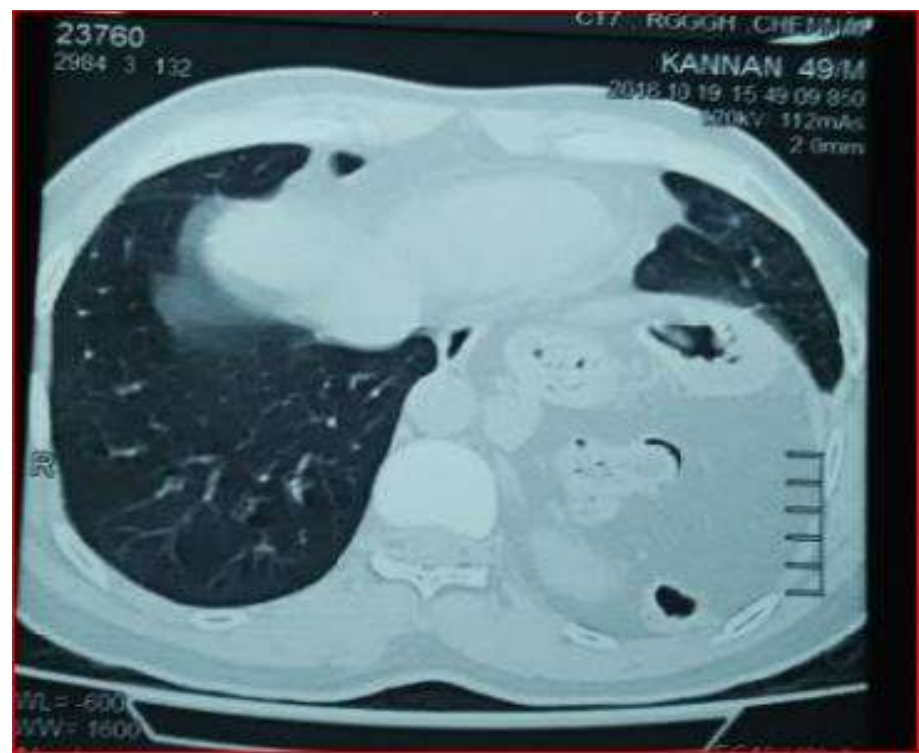

Fig. 2 Computed tomography showing Left diaphragmatic eventration with bowel and spleen as contents with a normal Lung parenchyma 